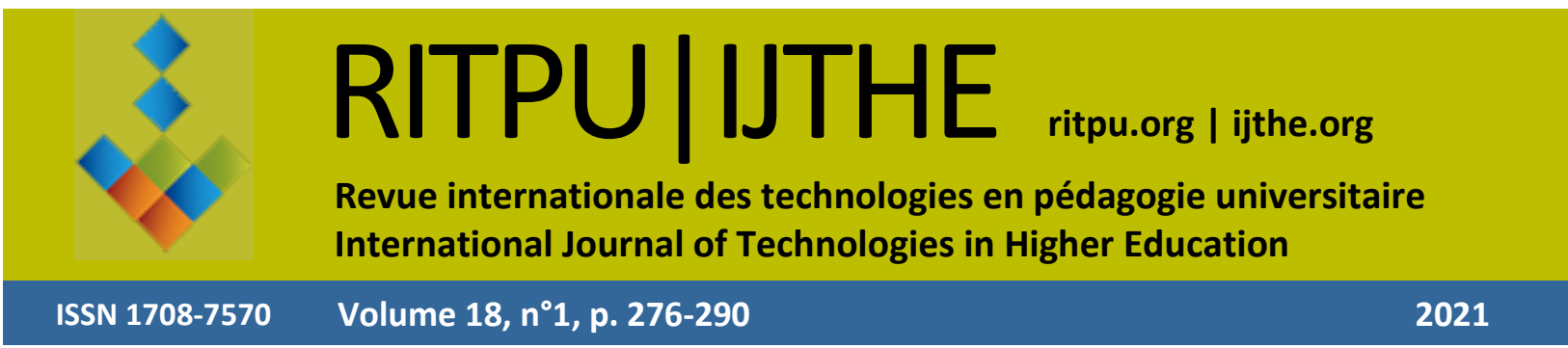

\title{
Évaluation des apprentissages en formation à distance : les situations authentiques à la rescousse
}

Anastassis KOZANITIS kozanitis.anastassis@uqam.ca Université du Québec à Montréal

\section{Evaluation of Learning Outcomes in Distance Education: Real-Life Situations to the Rescue}

https://doi.org/10.18162/ritpu-2021-v18n1-24

\section{Mis en ligne : 9 avril 2021}

\section{Résumé}

Cet article a pour but de proposer une réflexion pédagogique en vue de faire évoluer les pratiques évaluatives en contexte de formation à distance en milieu universitaire. L'évaluation en situation authentique est considérée comme un moyen pertinent pour mitiger les écueils et les craintes associés à l'évaluation à distance. Les propos prennent appui sur la littérature scientifique pour clarifier les concepts de situation authentique et présenter des critères et des conditions d'authenticité, tout en proposant des moyens efficients d'offrir de la rétroaction.

\section{Mots clés}

Évaluation authentique, formation à distance, authenticité, rétroaction

\section{Abstract}

The purpose of this article is to propose a pedagogical reflection with a view to developing assessment practices in the context of distance education in universities. Authentic assessment is considered a relevant way to mitigate the pitfalls and fears associated with online assessment. The comments draw on the scientific literature to clarify the concepts of an authentic situation, to present criteria and conditions of authenticity, while proposing efficient means of providing feedback.

\section{Keywords}

Authentic assessment, online learning, authenticity, feedback 


\section{Contexte}

La plupart des formations à distance en contexte universitaire impliquent une composante virtuelle qui permet, grâce aux technologies, la réalisation d'activités en modalité synchrone ou asynchrone. C'est par le truchement d'Internet que la présence physique isochronique et simultanée de l'enseignant et des étudiants dans un même endroit est remplacée par des rencontres en visioconférence, le téléchargement des documents préalablement téléversés sur un environnement numérique d'apprentissage (ENA), des outils d'interactions en différé comme les forums ou les wikis, et tout autre outil numérique dont la finalité est l'aide à l'apprentissage (Depover et al., 2007). La formation à distance existe depuis plusieurs décennies, avant même l'apparition des technologies évoquées (Bates, 2019). Ce qui est nouveau dans le contexte actuel de la crise sanitaire provoquée par la pandémie liée au coronavirus, c'est la contrainte temporelle pour faire basculer un cours en présentiel vers un cours à distance et le peu de préparation d'un grand nombre d'enseignants pour adapter leurs cours à cette modalité. Une modification rapide et parfois sommaire du passage vers un cours offert à distance peut provoquer certains malaises chez les enseignants, notamment en ce qui a trait à l'évaluation des apprentissages. Ce sont les enjeux inhérents à l'évaluation, en particulier sommative, qui inquiètent les enseignants et les administrateurs universitaires (Barras, 2020).

\section{Problématique}

Dans ce contexte, plusieurs se demandent comment contrôler les possibles actions inappropriées des étudiants, telles que le plagiat, la tricherie, la fraude et autres comportements non éthiques, lorsque la supervision directe devient une tâche compliquée à distance (Kier, 2014). Ces craintes sont sans doute légitimes de la part des enseignants qui ont la volonté et la responsabilité de s'assurer de la maîtrise par leurs étudiants des contenus enseignés. C'est d'ailleurs par souci d'équité que les conditions d'évaluation doivent être exemptes de comportements répréhensibles selon les politiques et les règlements qui régissent l'évaluation des apprentissages (Hewson, 2012).

Pour s'en assurer, certains établissements au Québec se tournent vers des solutions offertes par les outils technologiques de télésurveillance (Jung, 2020). Ainsi, avant de pouvoir répondre à un examen en ligne, les étudiants doivent installer un logiciel sur leur poste de travail qui donne accès au microphone et à la caméra pour que puissent être épiés leurs gestes, la localisation de la souris, les fenêtres ouvertes dans le navigateur, l'écran et les autres logiciels activés sur l'ordinateur. Mais cette solution d'examens télésurveillés n'est pas sans occasionner des irritants, le premier étant la réaction d'opposition des étudiants qui refusent d'installer ce genre de dispositif qu'ils considèrent comme une intrusion dans leur vie privée et une atteinte à la confidentialité numérique. De plus, le stress et l'anxiété ressentis devant une telle tâche d'évaluation sommative sont exacerbés par ces conditions, particulièrement dans un contexte de confinement comme celui que nous venons de subir. Il y a aussi les considérations techniques et logistiques, comme l'accès à un Internet à haut débit et à un ordinateur avec caméra, mais également à un environnement de travail calme, qui, si elles ne sont pas rencontrées, nuiraient aux principes d'équité évoqués précédemment.

La surveillance à distance n'est pourtant pas indispensable pour toutes les tâches d'évaluation. Il existe, outre les examens, d'autres moyens d'évaluer les apprentissages pour lesquels la prégnance de l'ensemble des considérations liées à la fraude universitaire est grandement réduite. Parmi ces moyens, l'évaluation en situation authentique s'avère une solution idoine et pérenne qui mérite qu'on s'y attarde (Janesick, 2006). L'évaluation en situation authentique, ou plus 
simplement l'évaluation authentique, est un processus de collecte d'informations à partir d'instruments basés sur des situations réelles, proposant des tâches complexes qui nécessitent des normes de performance et des critères spécifiques afin de fournir une rétroaction aux étudiants pour les aider à atteindre les objectifs d'apprentissage ou à développer les compétences (Huba et Freed, 2000).

Les études montrent que l'évaluation authentique peut avoir un impact positif sur la qualité et la profondeur des apprentissages et contribuer au développement d'habiletés cognitives d'ordre supérieur (Ashford-Rowe et al., 2013). De plus, celle-ci améliore l'autonomie, encourage l'engagement et suscite la motivation pour apprendre (Kearney, 2013). L'évaluation authentique favorise le transfert des apprentissages et procure des occasions d'appliquer des habiletés et des compétences disciplinaires et transversales, notamment la communication interpersonnelle, la pensée critique et la capacité de travailler en équipe (Swaffield, 2011).

Encore peu répandue dans les pratiques évaluatives en contexte universitaire québécois, l'évaluation authentique gagne néanmoins du terrain, particulièrement en formation à distance (Nizet et al., 2016). L'évaluation authentique peut prendre plusieurs formes, avoir des visées formatives ou sommatives et répondre aux différents besoins liés à l'évaluation (Dixon et Worrell, 2016). Il convient de rappeler que l'évaluation des apprentissages a, entre autres, pour buts de 1) vérifier l'atteinte des objectifs d'apprentissage ou le développement des compétences; 2) offrir de la rétroaction à des fins d'amélioration; 3) apporter des ajustements pédagogiques ou didactiques; 4) documenter les réalisations et le cheminement des étudiants en vue d'une certification (Scallon, 2015).

Dans le contexte de la formation à distance, l'évaluation authentique peut être réalisée avec ou sans l'utilisation d'outils numériques (Osborne et al., 2013). Elle peut aussi se faire de façon individuelle ou en équipe. Les études montrent que c'est la qualité de la conception de l'évaluation qui aura une influence sur la performance des étudiants plutôt que la modalité de réalisation de l'évaluation (à distance ou en présence). À ce sujet, plusieurs études rapportent n'avoir trouvé aucune différence significative sur le plan de la réussite ou des moyennes des notes des étudiants selon la modalité (Duesbery et al., 2015). De plus, la performance des étudiants ne semble pas être influencée par la préférence de la modalité des étudiants ou par leur degré d'aisance avec la technologie (Hewson, 2012). Ce dernier aspect nous semble important à mentionner, notamment dans le contexte de la pandémie, où la fermeture des établissements d'enseignement a exacerbé les inégalités numériques lors du passage au tout en ligne (Collin, 2020).

En dépit de ses nombreux avantages, il convient de souligner que certains défis peuvent limiter un usage plus répandu de l'évaluation authentique, particulièrement pour les cours du premier cycle universitaire. Les enseignants hésitent, car ils ont l'impression que la conception et la correction d'évaluations authentiques exigeront beaucoup de temps, d'efforts et de ressources intellectuelles (Grion et al., 2019). Ces évaluations peuvent également être perçues comme étant plus risquées (Dawson et al., 2019). De plus, elles exigent que les enseignants possèdent des connaissances disciplinaires étendues et fassent preuve de flexibilité cognitive pour superviser, confronter et guider adéquatement les étudiants vers des solutions pertinentes et rigoureuses sur le plan disciplinaire (Kohlmeier et al., 2020). Il est possible que la réaction initiale des étudiants en soit une de résistance et de crainte à cause de l'incertitude liée aux attentes et à la façon d'être notés (Fox, Freeman, Hughes et Murphy, 2017). Enfin, en dépit d'un important corpus faisant état de l'utilité et de la valeur de l'évaluation authentique, l'évolution vers cette pratique peut 
être entravée par un manque de clarté conceptuel de l'authenticité et de l'évaluation authentique (Bialystok, 2017).

Dès lors, cet article a pour objectif de proposer une réflexion pédagogique sur les manières d'adapter les pratiques évaluatives lors d'un passage vers la formation à distance en contexte universitaire, qui peut parfois devoir se faire dans l'urgence. Plus particulièrement, il a pour intention de faire état des principes généraux de l'évaluation en situation authentique, tels que soutenus par la littérature scientifique, d'étayer le concept d'authenticité et de mettre en lumière les éléments essentiels à sa mise en œuvre, comme reconnu par la communauté d'experts (Whitelock et Cross, 2012; Wiggins, 2011).

Précisons que l'étendue de cet article ne permet pas d'aborder l'ensemble des moyens, des méthodes et des outils relatifs à l'évaluation authentique. Cela dit, divers ouvrages spécialisés ont été publiés sur le sujet et peuvent guider les enseignants pour la planification de l'évaluation authentique (voir par exemple Duval et Pagé, 2013; Leroux, 2010a, 2010b, 2015; Prégent et al., 2009, chap. 5; Romainville et al., 2012; Scallon, 2015; Tardif, 2006). Par ailleurs, même si on a tendance à associer l'évaluation en situation authentique aux programmes de formation conçus par compétences, elle ne leur est pas exclusive (Talbot et Raîche, 2017). De ce fait, c'est le vocable «intentions pédagogiques », qui réfère à la fois aux compétences et aux objectifs d'apprentissage, qui sera désormais utilisé dans ce texte.

\section{Éléments conceptuels}

Cette section présente certains concepts et principes relatifs à l'évaluation authentique. On relate d'abord ce qui distingue l'évaluation authentique de l'évaluation qui a traditionnellement dominé les pratiques évaluatives en contexte universitaire, tout en proposant une série de critères et d'indicateurs d'authenticité. Puis, on aborde le rôle que peuvent avoir les différents axes de l'alignement pédagogique sur la perception des étudiants de la valeur de l'évaluation authentique. Enfin, on explore succinctement quelques solutions permettant de fournir de la rétroaction aux étudiants de façon efficace, notamment dans un contexte de confinement.

\section{Évaluation authentique}

Il y a trois objets d'évaluation à considérer: 1) l'évaluation des connaissances acquises; 2) l'évaluation de la capacité à agir dans une situation; 3) l'évaluation de la capacité de réflexion. Alors qu'est-ce qui distingue l'évaluation dominante de l'évaluation en situation authentique? L'évaluation dominante, c'est-à-dire celle qui a recours aux examens et aux tests, sert essentiellement à vérifier la maîtrise des connaissances, par la restitution ou la reproduction de celles-ci, très souvent de manière décontextualisée. L'évaluation en situation authentique sert plutôt à vérifier la capacité de résoudre des situations ou des problèmes complexes en ayant recours aux connaissances appropriées de façon efficiente, pertinente et contextualisée (Gulikers et al., 2004). L'évaluation authentique propose des tâches complexes représentatives de la réalité quotidienne ou professionnelle (Frey et al., 2012). Elle demande aux étudiants de réaliser une performance qui témoigne de leur capacité d'appliquer de façon significative leurs connaissances et leurs habiletés (Murphy et al., 2017). L'évaluation authentique est réaliste, elle favorise le jugement, la réflexion, la créativité et parfois l'innovation. Elle peut simuler le contexte d'une situation de travail ou du monde réel et promeut la consultation, la rétroaction et l'amélioration (Wiggins, 2011). 
Par ailleurs, Mueller (2009) propose de situer ces deux catégories d'évaluation sur un continuum plutôt que de les opposer de façon absolue; il suggère aussi de les considérer comme complémentaires. Les pratiques évaluatives peuvent ainsi se situer sur ce continuum d'authenticité selon cinq critères, dont les extrémités respectives sont les suivantes : 1) sélection d'une réponse - accomplissement d'une tâche; 2) contexte artificiel - contexte du monde réel; 3) rappel d'informations/reconnaissance - construction/application; 4) centré sur l'enseignant centré sur l'étudiant; 5) preuve indirecte - preuve directe.

Pour illustrer ces critères dans un contexte de formation à distance, prenons l'exemple d'une épreuve individuelle comportant des questions à choix multiples, réalisée en ligne avec le module «Test» sur un ENA comme Moodle. Pour le premier critère, cette évaluation se situe du côté «sélection d'une réponse ». Le contexte est plutôt artificiel puisqu'il est inusuel dans le monde réel qu'on nous demande de choisir parmi quatre options pour démontrer notre capacité à résoudre une situation. Cette épreuve sollicite la capacité de rappel d'informations ou de reconnaissance d'une solution. Elle est centrée sur l'enseignant, c'est ce dernier qui a structuré l'épreuve, choisi les questions, établi les choix de réponse et déterminé le format de remise. Pour ce qui est de la preuve d'apprentissage, elle est indirecte puisqu'il est difficile de connaître la part de chance ou le processus de la pensée qui aurait mené au bon choix de réponse.

La situation problème et la nature des tâches à réaliser sont considérées comme les éléments centraux de l'évaluation authentique (Gulikers et al., 2004; Murphy et al., 2017). Pour ces auteurs, la situation problème permet de faire le pont entre les savoirs théoriques et la façon dont ils peuvent être appliqués dans le monde réel. Il revient à l'étudiant de mobiliser et d'articuler l'ensemble des trois dimensions de connaissances, soit des connaissances déclaratives, procédurales ou conditionnelles, en vue de réaliser une tâche dont l'issue est incertaine, ce qui traduit le caractère complexe de la situation. Précisons que les connaissances déclaratives renvoient aux faits, aux concepts, aux idées, aux théories, aux théorèmes, etc. Les connaissances procédurales indiquent comment faire une tâche ou une praxis, il s'agit du savoir-agir. Les connaissances conditionnelles consistent à savoir quand utiliser les deux premières dimensions de connaissances.

Le tableau 1 présente les différences entre les situations du monde réel et celles du monde livresque (Wiggins et McTighe, 2005). Ainsi, la situation ou le problème à résoudre est considéré comme non authentique ou fragmenté pour les situations livresques, et authentiques ou complexes pour le monde réel.

\section{Tableau 1}

Distinctions entre situations livresques et situations réelles

\begin{tabular}{lcc} 
& Monde livresque & Monde réel \\
\hline Situation ou problème à résoudre & Non authentique ou fragmenté & Authentique ou complexe \\
Solution & Connue & Inconnue \\
Résultat souhaité & Inconnu & Connu \\
\hline
\end{tabular}

La solution, c'est-à-dire la façon de résoudre le problème ou la situation, est connue du côté livresque, inconnue du côté du monde réel. Enfin, le résultat souhaité, c'est-à-dire la réponse, est inconnu du côté livresque, tandis qu'il est connu du côté du monde réel. Pour illustrer cette distinction, prenons ces deux situations problèmes, présentées au tableau 2. 


\section{Tableau 2}

Exemples de situations problèmes théoriques et réelles

Situation problème $\mathbf{A}$

Situation problème $B$

On considère la fonction $f$ définie sur $\mathbb{R}-\{1,5\}$ par :

$$
f(x)=\frac{x^{2}}{2 x-3}
$$

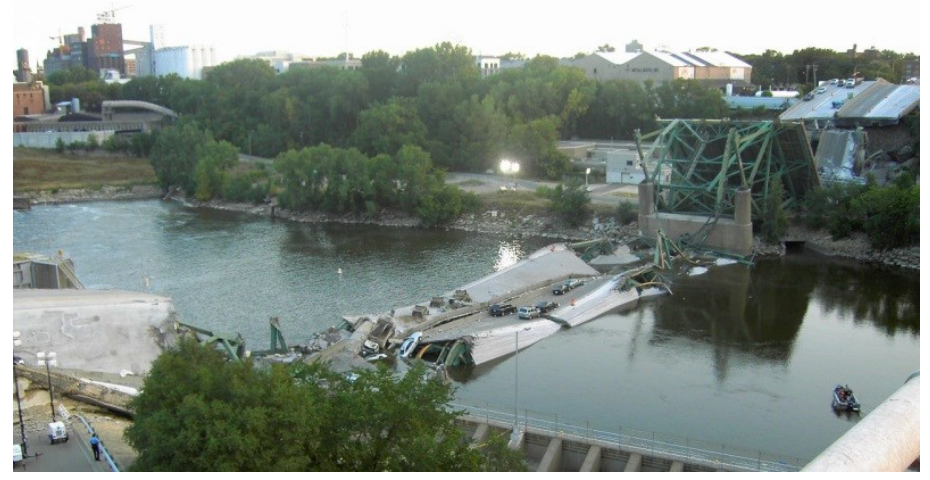

Source : Flickr, (C) Mike Wills, CC BY-SA

Tâche : déterminer une équation de la tangente $T$ à la courbe de $f$ au point d'abscisse 1 .

Tâche : concevoir un pont pour un emplacement géographique donné

Pour la situation $\mathrm{A}$, le problème à résoudre est fragmenté, c'est-à-dire qu'il est pris hors d'un contexte du monde réel, et l'on s'attend à une réponse unique. Pour sa part, la situation B est authentique et le problème à résoudre est complexe en raison du nombre de variables à prendre en considération pour proposer une solution adéquate. De la sorte, la façon de résoudre la situation B n'est pas connue d'emblée, plus d'une manière adéquate pourrait être proposée pour la résoudre. Pour ce qui est du résultat souhaité à la situation $\mathrm{B}$, il est connu d'emblée, on veut un pont qui ne s'effondre pas. En contrepartie, pour la situation A, la réponse (le résultat souhaité), ne doit pas être connue d'avance.

\section{Indicateurs d'authenticité}

Les situations et les tâches sont donc importantes puisque c'est principalement à travers elles que l'évaluation peut être considérée comme authentique. Cela dit, d'autres aspects peuvent également y contribuer, notamment le format de remise demandé (par exemple, une performance, un rapport, une vidéo), l'évocation d'un utilisateur final, c'est-à-dire la personne qui profitera du travail (un client, un patient, un consommateur, etc.), ou encore la finalité du travail. L'authenticité peut aussi renvoyer à la disponibilité des ressources, à la présence de collaborateurs, à la durée ou à la marge de négociation permise pour la réalisation du travail (James et Casidy, 2018). Ces aspects d'authenticité s'inscrivent dans le cadre conceptuel développé par Bosco et Ferns (2014) pour le contexte universitaire. Ce cadre comprend quatre critères d'authenticité, soit un engagement contextualisé de l'étudiant, un engagement cognitif d'ordre supérieur, une réflexion pour évaluer sa performance et, idéalement, une participation du milieu professionnel au processus d'évaluation.

Lorsque de telles conditions ne peuvent être remplies, on peut avoir recours à des évaluations écrites qui simulent le monde réel, comme des analyses de cas (Bédard et al., 1991), la résolution de problèmes (Bixler et Land, 2010), la création de cartes conceptuelles (Bai, 2013) ou la rédaction d'essais. La seconde dimension requiert que la tâche d'évaluation représente pour l'étudiant un défi sur le plan cognitif et mène à la réalisation d'une production ou d'une performance (Ashford-Rowe et al., 2013). De la sorte, le jugement de l'étudiant est sollicité afin de résoudre des problèmes, prendre des décisions et appliquer des connaissances, ce qui 
contribue au développement d'habiletés cognitives et métacognitives (Belland et al., 2017). En ce sens, l'évaluation authentique devrait mener les étudiants à établir des liens entre leurs connaissances antérieures et de nouvelles notions, entre les concepts théoriques et le monde réel, et à juger de la pertinence des arguments théoriques dans leur application pratique (Villarroel et al., 2018). La troisième dimension sert à encourager la réflexivité chez les étudiants. Une réflexion qui porte à la fois sur leur devenir professionnel et sur la qualité du travail réalisé (Tai et al., 2018). Ainsi, l'évaluation authentique les aide à se projeter au-delà de leur rôle d'étudiant pour anticiper les exigences du marché du travail et prendre les mesures nécessaires pour réussir (Nisula et Metso, 2019). Puis, en ayant d'emblée accès aux critères d'évaluation, les étudiants sont mieux à même de s'autoévaluer pour juger de la qualité de leur travail et de s'autoréguler (Bruijn-Smolders et al., 2016). Par ailleurs, d'aucuns sont d'avis que l'évaluation formative, en contexte d'évaluation en situation authentique, devrait faire partie des pratiques évaluatives des enseignants (Boud, 2010; Daly et al., 2010; Dixon et Worrell, 2016; Gikandi et al., 2011). Celle-ci donne la chance aux étudiants de constater leurs erreurs, de former leur jugement sur la qualité de leur travail et, en conséquence, d'améliorer leur performance.

Qui plus est, une synergie de ces trois dimensions d'authenticité offre plusieurs avantages. D'abord, les étudiants mentionnent que la réalisation de tâches en situation authentique peut avoir un impact sur la qualité et la profondeur des apprentissages (Strobel et al., 2013) de même que sur le développement d'habiletés cognitives d'ordre supérieur (Ashford-Rowe et al., 2014; Kozanitis, 2019). Ces tâches ont également l'avantage de promouvoir la confiance en soi, l'autorégulation et l'autonomie des étudiants ainsi que leur motivation et leur engagement scolaire (Kearney, 2013; Singaravelu et Reddy, 2020).

\section{Alignement pédagogique multiaxes}

$\mathrm{Du}$ point de vue de la didactique, le modèle de l'alignement pédagogique de Biggs et Tang (2007) insiste sur une démarche d'enseignement-apprentissage congruente entre les intentions de formation, le choix des activités d'enseignement-apprentissage orchestrées par l'enseignant et la façon d'évaluer les apprentissages. L'alignement peut se situer au niveau du cours et au niveau du programme, de sorte que les activités d'apprentissage proposées servent aux intentions pédagogiques du cours et du programme. De la même façon, les activités d'évaluation peuvent vérifier les intentions pédagogiques du cours ou du programme. Ce deuxième niveau d'alignement pédagogique se manifeste dans les programmes de formation soucieux de l'approche programme (Prégent et al., 2009).

Le modèle taxonomique développé par Bloom et ses collègues (1956), une référence fréquemment citée concernant les intentions d'apprentissage, organise ces dernières en trois domaines: le cognitif, l'affectif et le psychomoteur. Chaque domaine situe les intentions pédagogiques sur une échelle à complexité croissante. Par exemple, pour les intentions du domaine cognitif, le plus répandu en contexte universitaire québécois, les habiletés cognitives associées à chacun des échelons renvoient à la capacité de mémoriser, de comprendre, d'appliquer, d'analyser, d'évaluer et de créer (Anderson et Krathwohl, 2001). Initialement, une croyance largement répandue dans les milieux d'enseignement voulait que l'organisation hiérarchique des échelons soit cumulative et que le développement des habiletés cognitives associées à chaque échelon se doive d'être séquentiel. Ce que nous savons maintenant, c'est que le modèle taxonomique de Bloom postule que le développement des habiletés cognitives situées aux échelons supérieurs inclut le développement des habiletés des échelons inférieurs (Jensen et al., 2014). Les apprenants sont en quelque sorte astreints à la maîtrise d'une habileté inférieure 
pour maîtriser une habileté supérieure. Par exemple, il serait incongru d'appliquer un concept qu'on ne comprend pas, ou de créer un procédé ou un objet sans d'abord avoir analysé la situation qui requiert sa création.

S'inspirant de cette prémisse, les travaux d'Ajjawi et al. (2020) montrent que l'alignement pédagogique, tel que construit par l'étudiant, contribue à la perception d'authenticité. Ainsi, la valeur accordée par les étudiants aux tâches qui leur sont proposées détermine la perception de la valeur pour leur apprentissage. Plusieurs études ont montré que les aspects d'authenticité tels que perçus par les étudiants expliquent la plus forte variance de l'atteinte des résultats d'apprentissage (learning outcomes). À ce propos, Smith et Worsfold (2015) suggèrent que l'authenticité cognitive est toute aussi importante que la création d'un environnement d'apprentissage qui reflète le contexte physique du monde réel.

\section{Rétroaction}

La rétroaction est importante, peu importe le type d'évaluation. Elle permet aux étudiants de recevoir de l'information précise sur la qualité ou la pertinence de leur performance. La rétroaction comme pratique d'évaluation formative met à profit le cycle «performance, rétroaction, amélioration, nouvelle performance » évoqué par Wiggins (2011). En ce sens, elle est considérée comme un moyen efficace pour favoriser l'apprentissage et la réussite des étudiants (Hattie et Timperley, 2007). Par contre, son efficience est souvent remise en question, particulièrement lorsque la taille des groupes est élevée, le temps requis pour la réaliser lui étant corrélé. C'est la principale raison évoquée par les enseignants pour ne pas retenir cette pratique évaluative (Daly et al., 2010). Cela dit, certains moyens peuvent être mis en place pour mitiger cet écueil. À ce sujet, on peut considérer le choix de la source (l'enseignant, un étudiant ou une entité non humaine) et de l'objet (niveaux taxonomiques) sur lequel porte la rétroaction, les grilles d'évaluation descriptives ainsi que la rétroaction par procuration. Tous ces moyens visent à diminuer le temps requis pour produire la rétroaction, tout en ayant un impact positif sur la qualité des apprentissages.

\section{Sources et objets de rétroaction}

Il existe au moins trois sources de rétroaction, soit l'enseignant, l'étudiant et une entité non humaine (Moodle, par exemple), chacune ayant ses caractéristiques respectives. Le tableau 3 présente de façon succincte les caractéristiques des sources et le temps requis pour l'enseignant.

\section{Tableau 3}

Comparaison des sources de rétroaction

\begin{tabular}{lcc}
\hline Source de rétroaction & Caractéristiques & Temps requis \\
\hline Enseignant & $\begin{array}{c}\text { Contextualisée } \\
\text { Personnalisée }\end{array}$ & Considérable \\
& $\begin{array}{c}\text { Complémentaire } \\
\text { Eariable }\end{array}$ & Léger \\
Étudiant (pair) & Générique & Presque nul \\
Entité non humaine & Automatique & \\
\hline
\end{tabular}

L'enseignant peut donner à chacun de ses étudiants une rétroaction qui soit contextualisée et personnalisée. Par contre, le temps requis pour le faire peut être considérable, surtout lors de la formulation de commentaires. Les étudiants d'un même groupe peuvent à leur tour donner une 
rétroaction au travail d'un pair. Il est toutefois préférable de considérer cette source comme complémentaire, car la qualité peut être variable entre les individus. Enfin, l'entité non humaine peut offrir une rétroaction qui pour l'instant demeure générique; l'intelligence artificielle pourra éventuellement changer la donne. Néanmoins, elle libère le temps de l'enseignant, qui pourra se consacrer à d'autres tâches une fois le dispositif conçu.

L'objet sur lequel porte la rétroaction dépend de la tâche à réaliser. On peut croiser les intentions pédagogiques avec les dimensions des connaissances pour situer les tâches à l'intérieur d'une matrice afin d'aider à déterminer les sources pertinentes de rétroaction. Le tableau 4 présente la matrice à double entrée des sources de rétroaction. La rétroaction pour les tâches correspondant aux cellules de couleur bleu pâle peut être réalisée par l'entité non humaine. Cela libère l'enseignant et les étudiants, qui eux peuvent être la source de rétroaction pour les tâches représentées par les cellules de couleur bleu foncé.

\section{Tableau 4}

Sources de rétroaction pertinentes selon les dimensions de connaissances et les intentions pédagogiques

\begin{tabular}{|c|c|c|c|}
\hline \multirow{2}{*}{$\begin{array}{l}\text { Intentions } \\
\text { pédagogiques }\end{array}$} & \multicolumn{3}{|c|}{ Dimensions de connaissances } \\
\hline & Déclaratives & Procédurales & Conditionnelles \\
\hline \multicolumn{4}{|l|}{ Créer } \\
\hline Évaluer & & Enseignant & - Étudiant \\
\hline \multicolumn{4}{|l|}{ Analyser } \\
\hline Appliquer & & Entité & \\
\hline Comprendre & & non humaine & \\
\hline Mémoriser & & & \\
\hline
\end{tabular}

Le recours à des grilles d'évaluation descriptives offre également une certaine économie de temps pour la correction et la rétroaction (Cockett et Jackson, 2018). On retrouve souvent celles-ci sous forme de tableau qui contient les informations pertinentes permettant de juger de la qualité du travail réalisé (Côté, 2014). En ayant accès aux grilles d'évaluation ainsi qu'aux critères d'évaluation, les étudiants peuvent autoévaluer leur travail avant de le soumettre. Le fait de rendre les grilles d'évaluation accessibles contribue à abaisser le degré d'anxiété que peuvent éprouver certains d'entre eux vis-à-vis une évaluation authentique (Andrade et Du, 2005). Cela semble un avantage non négligeable dans le contexte anxiogène provoqué par la pandémie. De plus, ces grilles peuvent augmenter la confiance en soi (Lietchfield et Dempsey, 2015) et aident à rendre la correction plus objective (Prestidge et Glaser, 2000).

Il y a trois entités sur lesquelles peut porter l'évaluation des apprentissages, soit le produit, le processus et le discours. Le produit, c'est ce que les étudiants ont remis, c'est-à-dire l'artéfact, sur support physique ou virtuel de ce qu'ils ont fait, ou les performances réalisées. Le processus correspond à la manière dont ils ont fait ce qu'ils ont fait. Le discours met en lumière la justification de leurs choix, c'est-à-dire pourquoi ils l'ont fait de cette façon. Cette troisième entité est particulièrement intéressante dans le contexte de l'évaluation authentique puisqu'elle permet de vérifier si les étudiants ont utilisé les connaissances et les contenus du cours à bon 
escient pour résoudre la situation ou le problème complexe. Il s'agit tout simplement d'inclure cette entité dans la grille d'évaluation et de lui attribuer une pondération.

Enfin, la rétroaction par procuration consiste à rendre accessibles des travaux réalisés durant des sessions antérieures (Scoles et al., 2012). Cette pratique est bien appréciée des étudiants, car elle leur permet de se faire une idée concrète de la signification des critères d'évaluation (To et Carless, 2015). Les exemples aident à mieux comprendre ce qui constitue un travail de qualité et à appréhender la façon dont sera évaluée la qualité de leur travail.

\section{Dernières réflexions}

Les conditions sanitaires actuelles ont placé la plupart des enseignants dans une situation d'urgence pour l'adaptation de leurs cours en mode d'enseignement à distance. Cette transition abrupte et soudaine ne s'est pas produite sans causer un certain degré d'anxiété et des questionnements, notamment sur les façons d'enseigner et d'évaluer les apprentissages. Mais, il convient de paraphraser Albert Einstein qui prétendait que toute difficulté recèle une possibilité. Pourquoi ne pas utiliser alors la possibilité pour convertir cette calamité virale, qui s'est répandue et a entraîné la pandémie, en une occasion pour se diriger vers des pratiques pédagogiques proposant des tâches d'apprentissage et d'évaluation plus authentiques, contextualisées et qui mobilisent des habiletés cognitives d'ordre supérieur.

Nous tenons immédiatement à rassurer les enseignants sur les écueils et préoccupations mis en exergue en ouverture de l'article, particulièrement sur le temps requis et les ressources nécessaires pour l'implémentation d'une évaluation authentique. Nous l'avons maintes fois constaté, professeurs ou chargés de cours y parviennent à tout coup en quelques heures.

D'abord, le postulat d'inclusion subordonnée du modèle taxonomique de Bloom est la clé de voûte qui laisse entrevoir la possibilité de faire évoluer les pratiques évaluatives, notamment en contexte de formation à distance, qu'elle s'opère dans l'urgence ou pas. Il suffit dans un premier temps de modifier, le cas échéant, les intentions pédagogiques du cours pour qu'elles se situent dans les échelons supérieurs (soit analyser, évaluer et créer). Puis, compte tenu du principe d'alignement pédagogique, les pratiques pédagogiques s'ajustent pour en respecter l'ordre. Ce faisant, l'évolution vers des pratiques évaluatives en situation authentique prend alors tout son sens. Cela ouvre la voie vers des types, des méthodes et des outils d'évaluation nombreux et variés, pouvant mitiger les limites de l'évaluation dominante et répondre aux craintes suscitées par l'évaluation à distance (Boud et Molloy, 2013). La nécessité de contrer le plagiat en recourant aux outils numériques parfois dispendieux et impopulaires auprès des étudiants n'est plus un problème, puisque les solutions aux situations authentiques ne sont pas uniques.

Il convient également de mentionner que plusieurs universités dans le monde ont vitement offert un appui pédagogique au corps enseignant afin de le soutenir dans cette transition. Elles ont mobilisé experts en formation à distance, services pédagogiques, services audiovisuels et centres et équipes de recherche, tous ayant été fortement sollicités durant les semaines et mois qui ont suivi la désertion obligée des salles de classe. L'aide apportée pouvait prendre différentes formes (ateliers, formations, webinaires, etc.). À ce propos, le Centre de recherche interuniversitaire sur la formation et la profession enseignante (CRIFPE) a mis à la disposition des enseignants des enregistrements de webinaires qui apportent certaines réponses à leurs questionnements en temps de pandémie. 
En terminant, par souci d'une évaluation juste, cohérente et équitable des apprentissages à distance, l'évaluation en situation authentique se révèle une option alternative intéressante, pérenne et théoriquement bien fondée.

\section{Références}

Ajjawi, R., Tai, T., Le Huu Nghia, T., Boud, D., Johnson, L. et Patrick, C.-J. (2020). Aligning assessment with the needs of work-integrated learning: The challenges of authentic assessment in a complex context. Assessment \& Evaluation in Higher Education, 45(2), 304-316. https://doi.org/10.1080/02602938.2019.1639613

Anderson, L. W. et Krathwohl, D. R. (dir.). (2001). A taxonomy for learning, teaching, and assessing: A revision of Bloom's taxonomy of educational objectives. Longman.

Andrade, H. et Du, Y. (2005). Student perspectives on rubric-referenced assessment. Practical assessment, Research and Evaluation, 10, article 3. https://doi.org/10.7275/g367-ye94

Ashford-Rowe, K., Herrington, J. et Brown, C. (2014). Establishing the critical elements that determine authentic assessment. Assessment \& Evaluation in Higher Education, 39(2), 205-222. https://doi.org/10.1080/02602938.2013.819566

Bai, H. (2013). Using digital mapping tool in ill-structured problem solving. International Journal for the Scholarship of Teaching and Learning, 7(2), article 11.

https://doi.org/10.20429/ijsotl.2013.070211

Barras, H. (2020). Évaluer dans l'urgence : en repensant sa planification à l'aide des principes issus de la gestion de crises. Évaluer - Journal international de recherche en éducation et formation, (hors-série $\mathrm{n}^{\mathrm{o}} 1$ ), 17-24. http://journal.admee.org/...

Bates, T. (2019). Teaching in a digital age. (2e éd.). BC Campus. http://openlibrary-repo.ecampusontario.ca/...

Bédard, M. G., Dell'Aniello, P. et Desbiens, D. (1991). La méthode des cas : guide d'analyse, d'enseignement et de rédaction. Gaëtan Morin.

Belland, B. R., Walker, A. E., Kim, N. J. et Lefler, M. (2017). Synthesizing results from empirical research on computer-based scaffolding in STEM education: A meta-analysis. Review of Educational Research, 87(2), 309-344. https://doi.org/10.3102/0034654316670999

Bialystok, L. (2017). Authenticity in education. Dans G. W. Noblit (dir.), Oxford research encyclopedia of education. https://doi.org/10.1093/acrefore/9780190264093.013.168

Biggs, J. B. et Tang, C. (2007). Teaching for quality learning at university ( $3^{\mathrm{e}}$ éd.). McGraw Hill.

Bixler, B. A. et Land, S. M. (2010). Supporting college students' ill-structured problem solving in a Web-based learning environment. Journal of Educational Technology Systems, 39(1), 3-15. https://doi.org/10.2190/ET.39.1.b

Bloom, B. S. (dir.). (1956). Taxonomy of educational objectives. The classification of educational goals: Handbook I. Cognitive domain. David McKay.

Bosco, A. M. et Ferns, S. (2014). Embedding of authentic assessment in work-integrated learning curriculum. Asia-Pacific Journal of Cooperative Education, 15(4), 281-290. http://eric.ed.gov/?id=EJ1113553 
Boud, D. (2010). Assessment 2020: Seven propositions for assessment reform in higher education. Australian Learning and Teaching Council. http://ltr.edu.au/...

Boud, D. et Molloy, E. (2013). Rethinking models of feedback for learning: The challenge of design. Assessment \& Evaluation in Higher Education, 38(6), 698-712. https://doi.org/10.1080/02602938.2012.691462

Bruijn-Smolders, M. de, Timmers, C. F., Gawke, J. C. L., Schoonman, W. et Born, M. P. (2016). Effective self-regulatory processes in higher education: Research findings and future directions. A systematic review. Studies in Higher Education, 41(1), 139-158. https://doi.org/10.1080/03075079.2014.915302

Cockett, A. et Jackson, C. (2018). The use of assessment rubrics to enhance feedback in higher education: An integrative literature review. Nurse Education Today, 69, 8-13. https://doi.org/10.1016/j.nedt.2018.06.022

Collin, S. (2020, 31 août). Il est plus que temps de prendre au sérieux les inégalités numériques et scolaires. La Conversation. http://theconversation.com/...

Côté, F. (2014). Construire des grilles d'évaluation descriptives au collégial : guide d'élaboration et exemples de grille. Presses de 1'Université du Québec.

Daly, C., Pachler, N., Mor, Y. et Mellar, H. (2010). Exploring formative e-assessment: Using case stories and design patterns. Assessment \& Evaluation in Higher Education, 35(5), 619-636. https://doi.org/10.1080/02602931003650052

Depover, C., Karsenti, T. et Komis, V. (2007). Enseigner avec les technologies. Favoriser les apprentissages, développer des compétences. Presses de l’Université du Québec.

Dixon, D. D. et Worrell, F. C. (2016). Formative and summative assessment in the classroom. Theory Into Practice, 55(2). https://doi.org/10.1080/00405841.2016.1148989

Duesbery, L., Brandon, R. R., Liu, K. et Braun-Monegan, J. (2015). Transitioning to online courses in higher education. Distance Learning, 12(4), 7-15.

Dawson, P., Henderson, M., Mahoney, P., Phillips, M., Ryan, T., Boud, D. et Molloy E. (2019). What makes for effective feedback: staff and student perspectives. Assessment and Evaluation in Higher Education, 44(1), 25-36, https://doi.org/10.1080/02602938.2018.1467877

Duval, A.-M. et Pagé, M. (2013). La situation authentique : de la conception à l'évaluation. Chenelière Éducation.

Fox, J., Freeman, S., Hughes, N. et Murphy, V. (2017). Keeping it real: A review of the benefits, challenges and steps towards implementing authentic assessment. All Ireland Journal of Teaching and Learning in Higher Education, 9(13), 3232-3243. https://ojs.aishe.org/...

Frey, B., Schmitt, V. et Allen, J. (2012). Defining authentic classroom assessment. Practical Assessment, Research and Evaluation, 17, article 2. https://doi.org/10.7275/sxbs-0829

Gikandi, J. W., Morrow, D. et Davis, N. E. (2011). Online formative assessment in higher education: A review of the literature. Computers \& Education, 57(4), 2333-2351. https://doi.org/10.1016/j.compedu.2011.06.004 
Grion, V., Serbati, A., Felisatti, E. et Li, L. (2019). Peer feedback and technology-enhanced assessment as critical issues to foster student learning. Italian Journal of Educational Research, ( ${ }^{0}$ thématique, mai), 9-14. http://ojs.pensamultimedia.it/...

Gulikers, J. T., Bastiaens, T. J. et Kirschner, P. A. (2004). A five-dimensional framework for authentic assessment. Educational Technology Research and Development, 52(3), article 67. https://doi.org/10.1007/BF02504676

Hattie, J. et Timperley, H. (2007). The power of feedback. Review of Educational Research, 77(1), 81-112. https://doi.org/10.3102/003465430298487

Hewson, C. (2012). Can online course-based assessment methods be fair and equitable? Relationships between students' preferences and performance within online and offline assessments. Journal of Computer Assisted Learning, 28(5), 488-498. https://doi.org/10.1111/j.1365-2729.2011.00473.x

Huba, M. et Freed, J. (2000). Learner-centered assessment on college campuses: Shifting the focus from teaching to learning. Allyn \& Bacon.

James, L. T. et Casidy, R. (2018). Authentic assessment in business education: Its effects on student satisfaction and promoting behaviour. Studies in Higher Education, 43(3), 401-415. https://doi.org/10.1080/03075079.2016.1165659

Janesick, V. J. (2006). Authentic assessment. Peter Lang.

Jensen, J. L., McDaniel, M. A., Woodard, S. M. et Kummer, T. (2014). Teaching to the test... or testing to teach: Exams requiring higher order thinking skills encourage greater conceptual understanding. Educational Psychology Review, 26(2), 307-329. https://doi.org/10.1007/s10648-013-9248-9

Jung, D. (2020, 27 mai). Examens à distance : des universités québécoises tentées par la télésurveillance. Radio-Canada. http://ici.radio-canada.ca/...

Kearney, S. (2013). Improving engagement: The use of "authentic self-and peer assessment for learning" to enhance the student learning experience. Assessment \& Evaluation in Higher Education, 38(7), 875-891. https://doi.org/10.1080/02602938.2012.751963

Kier, C. A. (2014). How well do Canadian distance education students understand plagiarism? The International Review of Research in Open and Distance Learning, 15(1), 227-248. https://doi.org/10.19173/irrodl.v15i1.1684

Kohlmeier, J., Howell, J., Saye, J. et Brush, T. (2020). Investigating teacher adoption of authentic pedagogy through lesson study. Theory and Research in Social Education, 48(4), 492-528. https://doi.org/10.1080/00933104.2020.1751761

Kozanitis, A. (2019). Análisis de la dimensiones del compromiso cognitivo a nivel postsecundario desde una perspectiva socio-cognitivista. Revista Diálogo Educacional, 19(62), 1234-1252. http://doi.org/10.7213/1981-416X.19.062.AO02

Leroux, J. L. (2010a). L'évaluation des compétences au collégial : un regard sur des pratiques évaluatives [rapport PAREA]. Cégep de Saint-Hyacinthe. http://cdc.qc.ca/...

Leroux, J. L. (2010b). Comment des enseignants du collégial évaluent-ils les compétences? [article de vulgarisation]. Cégep de Saint-Hyacinthe. http://cdc.qc.ca/... 
Leroux, J. L. (dir.). (2015). Évaluer les compétences au collégial et à l'université : un guide pratique. Chenelière / AQPC.

Lietchfield, B. C. et Dempsey, J. V. (2015). Authentic assessment of knowledge, skills and attitudes. New Directions for Teaching and Learning, (142), 65-80. https://doi.org/10.1002/tl.20130

Mueller, J. (2009). Assessing critical skills. Linworth Books.

Murphy, V., Fox, J., Freeman, S. et Hughes, N. (2017). "Keeping it real": A review of the benefits, challenges, and steps towards implementing authentic assessment. All Ireland Journal of Teaching and Learning in Higher Education, 9(3), 3231-3243. http://ojs.aishe.org/...

Nisula, A.-M. et Metso, S. (2019). Factors fostering vocational students' workplace learning success in the real workplace environment. Journal of Education and Work, 34(6-7), 552-569. https://doi.org/10.1080/13639080.2019.1673884

Nizet, I., Leroux, J. L., Deaudelin, C., Béland, S. et Goulet, J. (2016). Bilan des pratiques évaluatives des apprentissages à distance en contexte de formation universitaire. Revue internationale de pédagogie de l'enseignement supérieur, 32(2). https://doi.org/10.4000/ripes.1073

Osborne, R., Dunne, E. et Farrand, P. (2013). Integrating technologies into "authentic" assessment design: An affordances approach. Research in Learning Technology, 21. https://doi.org/10.3402/rlt.v21i0.21986

Prégent, R., Bernard, H. et Kozanitis, A. (2009). Enseigner à l'université dans une approcheprogramme. Guide à l'intention des nouveaux professeurs et chargés de cours. Presses internationales Polytechnique.

Prestidge, L. K. et Williams Glaser, C. H. (2000). Authentic assessment: Employing appropriate tools for evaluating students' work in 21 st-century classrooms. Intervention in School and Clinic, 35(3), 178-182. https://doi.org/10.1177/105345120003500308

Romainville, M., Goasdoué, R. et Vantourout, M. (2012). Évaluation et enseignement supérieur. De Boeck.

Scallon, G. (2015). Des savoirs aux compétences. Exploration en évaluation des apprentissages. De Boeck.

Scoles, J., Huxham, M. et McArthur, J. (2012). No longer exempt from good practice: Using exemplars to close the feedback gap for exams. Assessment and Evaluation in Higher Education, 38(6), 631-645. https://doi.org/10.1080/02602938.2012.674485

Singaravelu, G. et Reddy, P. J. K. (2020). Effectivity of self-regulated learning in psychology among student-teachers of master of education. International Journal of Analytical and Experimental Modal Analysis, 12(4), 371-383. http://ijaema.com/...

Smith, C., et Worsfold, K. (2015). Unpacking the learning-work nexus: Priming as lever for high-quality learning outcomes in work-integrated learning curricula. Studies in Higher Education 40(1), 22-42. https://doi.org/10.1080/03075079.2013.806456 
Strobel, J., Wang, J., Weber, N. R. et Dyehouse, M. (2013). The role of authenticity in designbased learning environments: The case of engineering education. Computers \& Education, 64, 143-152. https://doi.org/10.1016/j.compedu.2012.11.026

Swaffield, S. (2011). Getting to the heart of authentic Assessment for Learning. Assessment in Education: Principles, Policy \& Practice, 18(4), 433-449. https://doi.org/10.1080/0969594X.2011.582838

Tai, J., Ajjawi, R., Boud, D., Dawson, P. et Panadero, E. (2018). Developing evaluative judgement: Enabling students to make decisions about the quality of work. Higher Education, 76(3), 467-481. https://doi.org/10.1007/s10734-017-0220-3

Talbot, N. et Raîche, G. (2017). Validation du cadre de référence PIEA des pratiques d'évaluation des apprentissages en classe dans une approche par compétences selon la perception d'étudiants du collégial. Revue des sciences de l'éducation, 43(3), 90-123. https://doi.org/10.7202/1050974ar

Tardif, J. (2006). L'évaluation des compétences : documenter le parcours de développement. Chenelière Éducation.

To, J. et Carless, J. (2015). Making productive use of exemplars: Peer discussion and teacher guidance for positive transfer of strategies. Journal of Further and Higher Education, 40(6), 746-764. https://doi.org/10.1080/0309877X.2015.1014317

Villarroel, V., Bloxham, D., Bruna, D., Bruna, C. et Herrera-Seda, C. (2018). Authentic assessment: Creating a blueprint for course design. Assessment \& Evaluation in Higher Education, 43(5), 840-854. https://doi.org/10.1080/02602938.2017.1412396

Whitelock, D. et Cross, S. (2012). Authentic assessment: What does it mean and how is it instantiated by a group of distance learning academics? International Journal of E-Assessment, 2(1), 9-14. http://ijea.org.uk/...

Wiggins, G. (2011). A true test: Toward more authentic and equitable assessment. Phi Delta Kappan, 92(7), 81-93. https://doi.org/10.1177/003172171109200721

Wiggins, G. et McTighe, J. (2005). Understanding by design ( $2^{\mathrm{e}}$ éd.). Association for Supervision and Curriculum Development. 